\title{
Filial Piety and Academic Motivation: High-Achieving Students in an International School in South Korea
}

\author{
Jonathan Tam \\ University of Oxford \\ United Kingdom
}

\begin{abstract}
This study uses self-determination theory to explore the mechanisms of filial piety in the academic motivation of eight high-achieving secondary school seniors at an international school in South Korea, resulting in several findings. First, the students attributed their parents' values and expectations as a major source of the students' understanding of filial piety responsibilities in their academic pursuit. Second, the participants found ways to justify the authoritative parenting they received through a reciprocal relationship, especially when their parents were autonomy-supportive. Third, the definitions of filial piety responsibilities varied, depending on the students' personal and familial idiosyncrasies.
\end{abstract}

KEYWORDS: Self-determination theory, Confucian-heritage cultures, filial piety, motivation, South Korea

Filial Piety in Confucian Heritage Cultures Self-Determination Theory Methodology

Researcher Ethics, Positionality, and Reflexivity

Findings: Students' Desire to Meet the Perceived Demands of Filial Piety

Discussion

Conclusion

Acknowledgements

Notes

References

Author Contact

The academic success of Confucian-heritage culture $(\mathrm{CHC})$ students has been well documented in multiple international academic assessments. ${ }^{1}$ Confucianism has historically influenced the education system and citizens of many nations such as China, Taiwan, South Korea, Japan, and Singapore in varying degrees. Additionally, immigrants to the West from $\mathrm{CHC}$ contexts have often demonstrated academic excellence, and some scholars have attributed the CHC students' success to their discrete cultural differences compared to students in host countries (Chao, 2000). Indeed, many studies have credited the academic success of $\mathrm{CHC}$ students to parenting, with the terms of $\mathrm{CHC}$ parentchild relationship being heavily influenced by filial piety norms (e.g., Chao, 2000; Chen \& Ho, 2012; Chen \& Wong, 2014; Chow \& Chu, 2007; Hui, Sun, Chow, \& Chu, 2011; Louie, 2004). 
This study explores the mechanisms of filial piety in academic motivation by answering the research question, "How do high-achieving $\mathrm{CHC}$ students describe the role of filial piety in their academic motivation?" While the heart of this study lies in uncovering the mechanisms of filial piety in academic motivation, what emerges secondary to that is also the tension between the CHC's collectivist and the Western individualist orientation in an age of globalization. Therefore, the study will also pay attention to understanding how CHCs are undergoing processes of connecting to the West and dealing with changing filial piety expectations.

In this paper, I first define filial piety and explain its relevance to academic motivation. I then justify how Self-Determination Theory (SDT) is applied in my conceptual framework. After explaining the case study method, addressing ethical concerns, and supporting my dual teacher-researcher positionality, I present my analysis of data and discuss how filial piety is intertwined with SDT's three basic psychological needs of autonomy, competence, and relatedness. I conclude with recommendations for further research.

\section{Filial Piety in Confucian Heritage Cultures}

Filial piety has historically claimed the position as the foundation of all good conduct in the CHC canon. In this paper, filial piety in South Korea is understood as the guiding principle over $\mathrm{CHC}$ patterns of socialization that dictates what is proper behavior for children, such as how to love and respect their parents, care for their parents, obey their commands, memorialize ancestors, care for their own bodies, continue the family line, and bring honor to the family (Ho, 1994; Lew, Choi, \& Wang, 2011).

Research on the role of filial piety in the context of modern Confucian societies has found filial piety to be both helpful and harmful to individual psychological well-being. Studies have found that filial piety may not be beneficial to children's development due to the positive correlation with parental emphasis on obedience, indebtedness to parents, impulse control, proper conduct, overprotection, harshness, and inhibition of children's self-expression, selfmastery and all-round personal development (Ho, 1994) as well as children's cognitive conservatism and rigidity (Yeh \& Bedford, 2003). More recently, researchers have found that filial piety can both positively and negatively affect intergenerational relationships in elderly care ( $\mathrm{Ng}$, Griva, Lim, Tan, \& Mahendran, in press), adolescent schooling (Li, Zou, Liu, \& Zhou, 2014), and psychosocial outcomes (Chen, 2014).

Filial piety expectations frame $\mathrm{CHC}$ parenting and parents' influence on their CHC children's approaches to schooling. Broadly speaking, CHC parenting has higher levels of parental involvement in academia (Chao, 1994), and healthy parent-child relationships are generally found to be positively associated with higher academic attainment (Phillipson \& Phillipson, 2012). Also, CHC parents 
prepare their children for success by facilitating the internalization of values such as the importance of academia as a means for social mobility (Chao, 2000), association of success with effort rather than ability (Hau \& Salili, 1991), academic excellence based on diligence, self-cultivation, and self-perfection ( $\mathrm{Li}$, 2003), and preference of the collectivist orientation over the individualist orientation (Jang, Reeve, Ryan, \& Kim, 2009). Similarly, Fuligni and Zhang (2004) identified that obligation to family by bringing honor and repaying parents for their upbringing, respecting parental authority, and being willing to sacrifice for the family was associated with higher levels of academic motivation among adolescents. Furthermore, Chen and Wong (2014) found that filial piety beliefs affect an adolescent's understanding of academic intelligence and achievement.

Given that filial piety norms frame how parents influence their children's expectations towards academic achievement, it can also create tension upon intergenerational relationships when these expectations are not met. Yeh and Bedford's Dual Filial Piety Model (2003; see also Chen \& Wong, 2014) theorize that the positive and negative effects of filial piety on children can generally be assessed by parenting styles that reflect reciprocity and authority. Reciprocal styles of parenting lead to the children (a) respecting and loving parents and (b) supporting and memorializing parents. It is largely beneficial in strengthening interpersonal relationships and lowering parent-child conflict frequencies. Conversely, authority-based filial piety is largely considered detrimental by leading the children to (a) suppressing themselves and (b) glorifying their parents. It accentuates hierarchy and submission, leading children to comply with their parents' physical, financial, or social terms without their own regards. In other words, filial piety can manifest in both congenial and in authoritative ways.

The type of filial piety has clear implications on academic motivation and achievement. In studies testing filial piety and academic motivation and achievement, Chen and Wong (2014) found that reciprocal filial piety is directly linked with academic achievement. Conversely, Chen and Ho (2012) found no direct link between authoritative filial piety with academic achievement. In this study, I explored whether the participants' accounts of the extent of their filial piety affecting their academic motivation were related to their reciprocal or authoritative relationship with their parents.

In Park and Kim's (2015) comparative study of Koreans in different geographical settings, filial piety norms of family care and support, along with respect for parents, were found to remain as the intergenerational norm for Koreans. However, they found that filial piety's practices erode with major sociocultural and economic changes, such as migration. As a result, the next generation in their study developed a "bystander" attitude that sows the seed for family conflict. Clearly, the Western individualist orientation can come in conflict with the collectivist orientations of filial piety. Then, the question arises: "How does filial piety induce tension over academic expectations for students who have had significant exposure to so-called Western values by being educated in an international school but remain in South Korea? 


\section{Self-Determination Theory}

In addition to its conceptual focus on filial piety, this study applies SelfDetermination Theory (SDT: Jang et al., 2009; Ryan \& Deci, 2004) as the conceptual framework on how the data were collected, coded, and analyzed. SDT contends that all individuals have intrinsic motivation and a desire for selfdetermination. It maintains there are three essential psychological needs in the forms of competence, relatedness, and autonomy that are necessary for ongoing psychological growth, integrity, and well-being. These psychological needs, while useful as discrete analytical categories, are also intertwined and interrelated.

Competence refers to the sense of confidence and effectiveness in one's ability throughout the enduring interactions with the social context. This need for competence leads people to pursue challenges that are optimal to their capabilities and to persistently engage to maintain or improve those capabilities through activity. It does not refer to an attained skill or capability, but to a felt sense of confidence.

Relatedness refers to the sense of feeling connected to others. This includes caring and being cared for by others while having a sense of belonging with other individuals and one's community. This need to be in relation with others is not concerned with the attainment of various outcomes or a formal status but places primacy upon the psychological sense of being in a secure relationship with others.

Autonomy is concerned with the perceived origin of one's own behavior and acting from interest and integrated values. When autonomous, even when actions are influenced by outside sources, individuals experience their behavior as an expression of the self as long as they agree with the outside influences. Autonomy should not be confused with independence, which means one does not rely on external sources or influences, since one may agree to follow the directions or opinions as long as it is congruent with the self. Similarly, it is possible for one to comply or conform to the directions or opinions in a manner without autonomy.

Previous studies have used the SDT framework to investigate the role of filial piety in academic motivation. Chow and Chu's (2007) survey study found that Hong Kong Chinese students seek to fulfill their filial obligations through academic achievement as a means to repaying their parents and honoring their family. Their students identified four key concepts of filial piety in the study: "selfsacrificing obedience," "caring for parents," "respecting parental intervention," and "upholding family honor." Hui et al. (2011) found that filial piety had the strongest effect on a student's competence, followed by relatedness and autonomy. Pan, Gauvain, and Schwartz (2013) found that parental collectivist orientations both positively and negatively affected adolescent autonomy development. While contributing to the knowledge of filial piety's role in academic 
motivation, these studies adopted reductionist approaches to understanding filial piety and academic motivation, and they all asserted the need for qualitative studies to identify relationships in depth. For example, while collectivist culture operates as a "double-edged sword in relation to Chinese adolescents' autonomous motivation" (Pan et al., 2013), why this was the case remained unclear (also see Jang et al., 2009). This paper explores the knowledge gap regarding filial piety mechanisms in academic motivation.

\section{Methodology}

This research adopted a multi-case study approach by collecting data in 2012 through participant observation and in-depth semi-structured interviews with eight high-achieving South Korean international school students attending Happy Valley International School (HVIS, pseudonym). HVIS is a K-12 nondenominational Christian international school located in one of the satellite cities of Seoul, the capital of South Korea. The sample consisted of five female and three male participants. These students were 18 or 19 years of age at the time of the study and were in their senior year at HVIS. Given that the legal age for adults in South Korea at the time was 20 years old, consent forms were acquired from both the students and their parents. The students involved in the study were identified to be high-achieving by their membership in the National Honors Society (NHS) determined by their superior grades. Access only to the highachieving students was granted by HVIS for this study.

Given South Korea's strong Confucian tradition, the students are within the definition of a suitable population for qualitative analysis (Lew et al., 2011). However, like most qualitative research, given the small and exploratory nature of the study, it is necessary to recognize that the participants in this study are not representative of all South Korean high school students. The study's participants are a unique group of upper-class students who have been significantly influenced by both South Korean and, broadly speaking, Western sociocultural values given their upbringing (e.g., years living abroad) and experience with international school education (see Table 1). They are all ethnic Koreans who are linguistically and culturally proficient in both English and Korean. While English was the primary language used to conduct the study, Korean was used on occasion to communicate words and concepts that do not exist in English. ${ }^{2}$

Semi-structured interviews were conducted privately after school in classrooms. To ensure case diversity, sequential interviewing techniques were employed (Small, 2009) by adjusting the semi-structured interviews to explore different themes emerging within the students' narratives of how they attributed their academic motivation to filial piety. All interviews were tape-recorded and transcribed verbatim ${ }^{3}$ with the participant identified by a pseudonym. 
Table 1

Demographic information of participants

\begin{tabular}{|c|c|c|c|c|c|}
\hline \multirow[b]{2}{*}{ Name } & \multirow[b]{2}{*}{ Age } & \multirow[b]{2}{*}{ Sex } & \multirow[b]{2}{*}{ Nationality } & \multicolumn{2}{|l|}{ Number of years } \\
\hline & & & & Living in the West & In a Western school system \\
\hline Betty & 19 & $\mathrm{~F}$ & U.S.A. & 4 & 5 \\
\hline Jessie & 18 & $\mathrm{~F}$ & S. Korea & 3 & 2 \\
\hline Kit & 18 & M & S. Korea & 7 & 12 \\
\hline Curtis & 19 & M & S. Korea & 6 & 12 \\
\hline Cindy & 18 & $\mathrm{~F}$ & S. Korea & 6 & 10 \\
\hline John & 18 & M & Dual & 4 & 6 \\
\hline Julia & 19 & $\mathrm{~F}$ & S. Korea & 5 & 5 \\
\hline Mary & 18 & $\mathrm{~F}$ & Dual & 6 & 7 \\
\hline
\end{tabular}

Note: Dual $=$ both U.S.A. and South Korea

Each participant was conceptualized as an independent case for a total of eight cases for within-case and cross-case analysis (Yin, 2013). I first performed within-case analysis using the SDT framework to allow the themes to emerge. I then applied cross-case analysis to compare and contrast the findings between different cases. By comparing and contrasting the cases, common and divergent themes emerged. Guided by the study's conceptual framework of SDT, coding categories were developed and refined on an ongoing basis.

\section{Researcher Ethics, Positionality, and Reflexivity}

Clearance to conduct the study was given by the school administration upon their review of the interview questions and consent forms. I then recruited student participants on an understanding that no remuneration or negative consequences would result from their participation. These details were clearly stated in the consent form provided for both students and parents, and its signing was required before interviews began.

At the time of the study, I was employed as a faculty member at HVIS. Teaching the International Baccalaureate (IB) provided me the opportunity to establish rapport with most of the upperclassmen in the NHS for two years. Several important factors influenced my understanding of the participants' interviews. First, being ethnically Chinese gave me insight into the sociocultural nuances of CHCs and the potential of filial piety to affect academic motivation. Second, being born and raised in Canada provided an understanding of how socalled Western sociocultural values may influence an international student's 
interpretation of filial piety. Third, teaching in that school system in South Korea for five years afforded me an understanding of the students' and parents' sociocultural context.

I acknowledge that the same experiences that were beneficial in providing insight could function as a liability leading to biased judgments regarding research design and the interpretation of findings. Furthermore, an anticipated limitation will be the phenomenon referred to by Maxwell (2013) as participant reactivity, when interviewees experience difficulty adjusting to my changing roles from teacher to researcher. I took several steps to mitigate these issues. First, I made clear to the student participants when I was a teacher and when I was a researcher. Second, I committed to critical self-reflection by journaling and dialoguing with professional colleagues and academic advisors on this topic. Third, buffering several years before publication provides the objective space to account for my personal relationship with the students.

\section{Findings: Students' Desire to Meet the Perceived Demands of Filial Piety}

The study found that the participants had different understandings of the social responsibilities that filial piety demands of them. Nonetheless, there were two that all participants agreed upon: to love and care for parents and to make parents happy or proud. In addition to describing a highly reciprocal relationship, one of love and care, Julia further expressed the need to provide love and care for her family as a filial daughter: "Because I have to pay back her money (laughs) and show more love. Because I have been so busy working on schoolwork, I haven't able to pay attention to my mom or my family." When probed further on how this love and care is communicated, Julia replied, "By doing my schoolwork (laughs). By asking her to spend time together (pause) and that's how we express our love."

In addition to providing love and care for their families, all eight participants also expressed their desire to make their parents happy or proud.

Betty: Just for my parents, you know. In Korea there are a lot of pressures in education. Although my parents would certainly say to me that going to a good college doesn't make them happy as much as if I behaved in the right way. But...just as a Korean daughter, I think it was kind of an unconscious pressure. Or it kind of felt like a duty...if I go to a good college, that will make them happy. Before coming to this school, I didn't study at all and I kind of hurt them a lot.... So, because of that, I thought studying hard would make them happy, or just redeem myself.

The participants expressed other qualities of filial piety beyond the two common ones. Four participants stated being respectful to their parents as a way of fulfilling filial piety. When discussing showing respect to his father, Curtis 
believes that given the parents' sacrifice, there are lines that must not be crossed.

Curtis: I think all dads want a certain amount of respect from their children. They work hard but....I should respect him because he does provide for me. And l've experienced this with my dad... like in $9^{\text {th }}$ grade I slipped out a few words that I shouldn't say to my dad and I got into big trouble.... I was sleeping at like 3 o'clock in the morning and he turned on the lights.... don't know it just slipped out and then I got into trouble for saying bad words.... He's really cool and everything but I don't know what's going to happen if I intrude his like...you know his res- (abrupt stop).... I can't even imagine myself cursing my dad.

Some participants also alluded to the fact that their parents were role models for them. Cindy referred to her mother's exemplary work ethic in studying for her doctorate despite working on it for a while. "Yeah...even though she's kind of old, she still tries really hard to pursue her dreams that have been forgotten for a long time."

Three participants expressed the provision of financial support to parents as a filial responsibility. They claim this motivates them academically because having good grades is synonymous with finding a lucrative career:

Jessie: If a person really wants to do hyo-do (filial piety in Korean) to their parents, then they would want to be successful in their lives and make a lot of money to fulfill the financial part of their lives and for their parents. So I think it's not directly correlated but one of the ways to do hyo-do is to make your parents be...(long pause) uh...(laughs) financially okay and that would be by getting a good job and good grades.

Johnny: Our family isn't that rich and I understand how much my parents sacrificed in order to give me this opportunity to have one of the highest quality of education our country can offer. I mean, the world can offer...IB and international school. I appreciate it and I understand how much of a sacrifice they had to make....I'm still a teenager so I cannot pay back the money and I cannot take them to like the cruise ship and make them feel happy.

To put Johnny's statement in context, HVIS cost approximately $\$ 30,000$ USD annually at the time of the study. The fact that the parents of all the participants were willing to invest in their education communicates that they made their children's education a top priority in life. As a response, some participants desired to do well in school so they could repay their parents' investment.

While it is clear that the students attribute their academic motivation to filial piety, it is a comparatively weak motivator to their desire for autonomy. Seven of the participants expressed that their responsibilities of filial piety come after their desire for autonomy. Julia maintained that her pursuit of dreams comes before being a hyo-nyo (filial daughter) and she gets to choose her career, "I know that as a daughter, I should care for my mom because she raised 
me and took care of me. But I have my own dream... and I have my own greed (laughs)."

While most participants preferred to keep their autonomy, Johnny desired to surrender his autonomy to put his parents' preference first.

Johnny: But as a teenager the best thing I can do is showing them that whatever they have sacrificed wasn't just a piece of trash. Wasn't just worth nothing. I just want to show them that it was a very good investment and a very good sacrifice and was worth it and make sure I show them that I can grow much bigger out of what they provided me by myself. So showing that in my report cards and school reports can be one of those best ways to show that to my parents... right now I want to be a lawyer... that's one of my parents' preferences.

Despite wanting to impress his parents, it does not mean his pursuit was a wellresearched and informed decision. From my interactions with Johnny as a teacher, it was clear he did not know what a career in law entails. For example, during free time in class one day, Johnny was surprised when he came across the online contents of the meme website "Buzzfeed" where a lawyer posted a list of job characteristics reflecting how monotonous a career in law was and how it is not as dramatic as in movies. Nonetheless, Johnny was determined and still went on to pursue an education in law after graduating from HVIS.

While many students attributed their pursuit of education to filial piety, some make the argument that it is not the only way to be filial.

Jessie: Yeah, my dream would be my first motivation and....If thinking about the expectations from parents and hyo-do separately then...the expectations from parents be about second or third, including like expectations from friends and teachers.... don't think it's my number one motivation force, but rather like takes about $20 \%$ of my overall motivation.

Cause I can perform hyo-do in other ways, not just through school [success].

Furthermore, some stated that doing well in school is not motivated merely by filial piety reasons but it is aligned with their own desire for success. For example, Kit stated, "I have my own personal motivations as well and I want to argue that sometimes it has much more significant role (than filial piety)."

With different definitions of what filial piety entails, participants had varying answers when asked whether they perceived themselves to be filial or not. Four of the participants believed they are not filial but for different reasons. Betty cites her being disrespectful to her parents as a reason for being unfilial. "Regarding my behaviors recently, I'm definitely not filial because l've acted very selfishly and considered my other duties as important as family duties. I recently acted very arrogant, so I wouldn't say that I'm filial." Ironically, Betty's arrogance was precisely the result of high academic attainment in being admitted into an Ivy League university. Curtis cited financial reasons for him being unfilial, "No, at the moment I spend all my family's money but do not provide anything for them. I 
also fight a lot with my parents." Yet Kit cites his reason for being unfilial as not exceeding expectations:

I would like to define being a filial son as being a person who always behaves above expectations towards parents. However, I would not call myself as a son who behaves above expectations. However, at the least, I think I uphold the expectations and responsibilities expected of me.

Similarly, the participants who believed they are filial provided different reasons. Cindy expressed loving and fulfilling responsibilities as being filial, "Because I love my parents more than anything in this world and I am always grateful for what they have done for me. I always try to fulfill my duties as a daughter." Mary expressed similar sentiments that loving and respecting her parents as fulfilling filial piety,

Yes. I do what they expect of me, and beyond that. I respect them from the bottom of my heart and have my parents as my role models and try to be like them. Above all, my love towards them is undeniable.

From their accounts, it is clear that the students attributed much of their academic motivation to filial piety. It is also evident that a close parent-child relationship strengthens the students' desire to fulfill their perceived filial piety responsibilities. Specifically, loving and caring for their parents and making their parents proud are common themes in all the interviews. Furthermore, it is largely consistent from their accounts that academic competence as a means to a successful and lucrative career along with maintaining a close and respectful relationship is at the top of their main responsibilities. However, for some students, success in their education did not result in their self-perception of being filial as there are other expectations, some unrelated to schooling. A reason for this is that filial piety responsibilities are mostly ancillary to their pursuit for autonomy.

\section{Discussion}

There is an intricate relationship among competence, relatedness, autonomy, and filial piety in affecting academic motivation from the participants' narratives. The lack of a uniform definition of what it means to be filial made it difficult to clearly identify its role in academic motivation. Furthermore, even in cases where filial piety was directly referred to as an academic motivator, most participants maintained that it remained secondary to SDT's competence, relatedness, and autonomy. These findings lead to three points of analysis:

1. Parental transfer of filial piety values and expectations,

2. Reconciliation of authoritarian parenting with reciprocity, and

3. Filial piety as an academic motivator. 


\section{Parental Transfer of Filial Piety Values and Expectations}

From the narratives of the participants, it was clear that filial piety values and expectations from the participants' parents affected their behavior. These values and expectations were communicated both tacitly and overtly at a young age through high parental involvement in schooling. For example, the participants listed factors that included setting up the home environment, paying expensive tuitions for HVIS and hakwons (tutor center or cram schools), and monitoring and disciplining (especially when young). Most participants mentioned these reasons for their academic success, facilitating a snowball effect of confidence in their competence. Furthermore, all the students were acutely aware of the high costs of their international school and other education supports. All these served to communicate and transfer parental values and expectations on education.

Some participants indicated gendered norms on the parental participation in their children's schooling with the mother more involved in their schooling. Often, the mother not working increased her involvement at school. For example, Curtis made several references to his relatively relaxed relationship with his father to contrast his perpetual conflicts with his mother over school.

Curtis: We talk. But it's more about sports, man stuff, cars... He doesn't really care about my education.... If I'm going to UCLA or NYU, he's just like, "Go wherever."...my mom...her concern for me I guess is an expression of love. Maybe it's just all up in my head but my mom cares about what college I'm going to a lot. She always asks me "Where are you going to go?" It's annoying. At the same time, I can feel her....She expects a lot from me, I think....I guess they already expect me to become successful when I grow up and...just by saying that in the future I'm...going to be successful [she] exerts pressure on me.

In Curtis's statement provided above, he highlights the gendered roles of parenting in South Korean society found in other studies (Kim \& Hoppe-Graff, 2001). Specifically, the societal norms for South Korean fathers are to focus on work while the mother takes care of the home and the children's education. In fact, there is the slang in Korean called "chimat baram," literally meaning "skirt wind," to illustrate the gust of wind brought by hurried Korean mothers rushing to address their children's needs. This pressure exuded by Curtis's mother built up over time and became extremely apparent during his senior year college applications. The participants rarely referred to their fathers when discussing schooling pressures. Accounts like Curtis's suggest that much of the filial piety values and expectations over schooling come from the mother in the South Korean context. 


\section{Reconciliation of Authoritarian Parenting with Reciprocity}

All my participants maintained that the desires to love, care for, and make their parents happy were core tenets of filial piety. In addition, all participants expressed that parents were involved at home and felt they were loved and cared for by their parents. Even though there may be occasional tensions from authoritative parenting, there was a consistent desire for reciprocity.

Despite the parents sometimes coming across as authoritative, most of the participants were able to reconcile their parents' authoritative style as a form of reciprocity given their academic excellence. Central to this reciprocity was the fact that all the parents encouraged autonomy while simultaneously demanding academic excellence. For example, Mary expressed:

I guess doing what I want to do is the most important thing because my suffering from doing what I don't want to do is going to displease my parents because a parent's main goal for their children is for them to live a happy life.

Cindy resonated similar expectations, "They told me that they want me to pursue my dreams." By stating that the pursuit for academic excellence is synonymous with their quest for autonomy, the participants were able to align their filial piety values with their high parental expectations.

\section{Filial Piety as an Academic Motivator}

This study is consistent with prior findings of filial piety values being a weaker academic motivator relative to autonomy, competence, and relatedness (Hui et al., 2011). One main reason is because the interpretation of filial piety responsibilities varied by the individual. This could be explained when considering familial and personal factors on how one understands filial piety responsibilities.

Familial factors influenced the participants' understanding of what filial piety in schooling entails. The primary source of influence was from the parents. Other familial factors can include extended family influences and how the family (and the mother in particular) reacts to the peer pressure from fellow parents in the high pressure South Korean context. Naturally, parental and familial idiosyncrasies such as socioeconomic status, parental profession, parental gender roles, values, expectations, and parenting methods are important.

Personal idiosyncrasies are also a factor influencing the participants' understanding of filial piety responsibilities. The different interpretations of filial piety can only be partially attributed to the degree of Westernization of the individual or the family given the comparatively more expressive and congenial Western parental-child relationship. For example, Kit felt a distinct calling to the 
medical field to find a cure for his brother's ototoxic condition, and he cites that to be an important motivation for him to enroll into a medical program. In another example, contrary to the other accounts where the participants worked hard to appease their parents, Betty wanted to catch her parents' attention due to their lack of expectations for her.

Betty: My parents always kind of let me do what I wanted to do. They didn't expect a lot from me. Maybe that's why I wanted to do better. They always said, "I'm always satisfied by what you want to do."

Different familial and personal factors can affect one's degree of drive and direction of filial piety's influence on academic motivation. While this analysis separates familial and personal factors into discrete categories, they are naturally intertwined as familial factors inevitably influence the individual and vice versa. With variance in definitions and expressions of the filial piety concept, the manifestation of associated behavior attributable to it will also vary accordingly.

\section{Conclusion}

The relationship between filial piety and the basic psychological needs of autonomy, competence, and relatedness is intricate and intertwined. First, parental values and expectations during the schooling process were a major source of the students' understanding of filial piety responsibilities in their academic pursuit. Nonetheless, while filial piety serves as an academic motivator, the participants place it as a lower priority than the other three SDT needs of competence, relatedness, and autonomy (Hui et al., 2011). Second, the participants found ways to justify the authoritative parenting they received through their desire and actualization of closely bonding with the parents. This explains how the students were able to reconcile the "double-edged sword" (Pan et al., 2013) of collectivist culture (see also Chen \& Wong, 2014; Chow \& Chu, 2007). Particularly if the parents were autonomy-supportive, filial piety flourished since children believed that their parents wanted them to pursue their dreams. Third, the definitions of filial piety responsibilities varied by the student. The participants' accounts of how filial piety motivated them depended largely on idiosyncratic familial and personal factors. The accounts in this study help explain why it is difficult to pin down the extent that filial piety serves as an academic motivator because of the complex human factors that reductionist studies cannot adequately account for. This is especially the case for this study's sample where traditional collectivist values are being eroded in an age of globalization and migration (see also Park \& Kim, 2015).

This study carries implications for educators in multicultural settings. Filial piety expectations can lead to immense pressure for students to succeed academically. By understanding filial piety expectations, teachers can begin to consider how to alleviate or leverage such pressures. For example, teachers and administrators can come up with more effective means of working with $\mathrm{CHC}$ 
parents and students on how to alleviate stress induced by filial piety expectations by improving parent-child relationships. The findings suggest that encouraging authoritative parents to be more autonomy-supportive and building a congenial relationship can be effective. In essence, this knowledge equips educators to be more culturally fluent in dealing with their $\mathrm{CHC}$ students and parents.

There are several limitations in this study. First, there is a clear selection bias in that all the students interviewed are academically competent. It is therefore not particularly surprising that the participants have successfully aligned filial piety expectations given their academic motivation and success. What happens to the child-parent relationship if the parents demand high academic competence but do not encourage autonomy? How will the children's failure to meeting these academic expectations exacerbate tensions?

Second, the participants' unique backgrounds may frame the participants' understanding of filial piety. The high-achieving participants in this study come from a high socioeconomic status given that they can afford to attend an expensive international school. This is consistent with previous findings (e.g., Li et al., 2014) that high socioeconomic status and parental education are often predictors of reciprocal filial piety relationships among my participants. Furthermore, these students have experienced significant influences from the West given their international school education ${ }^{4}$ and time living abroad. Accounts from native South Korean students with less Western individualist influence and support may tell a different story.

Finally, the South Korean interpretation of filial piety may have its specific nuances compared to other $\mathrm{CHC}$ cultures. Studies comparing South Korean filial piety with other $\mathrm{CHCs}$ will contribute to understanding the differences between these contexts. Further studies that address these limitations will deepen our understanding of filial piety and its role in academic motivation.

\section{Acknowledgements}

I am grateful to Ron McGraw, Susan James, Colin Mills, and the anonymous reviewers for their insightful guidance and valuable feedback. An earlier version of this paper was presented at the Ethnography in Education Research Forum in February 2013 (Philadelphia, USA) and the Comparative and International Education Society in March 2013 (New Orleans, USA).

\section{Notes}

1. See results in the Trends in International Mathematics and Science Study, Progress in International Reading Literacy Study, and Program for International Student Assessment. 
2. At the time of the study, I was able to understand Korean terms and concepts at a rudimentary level. For more complicated terms, students were able to translate and elaborate on the spot.

3. While most of the students are fluent in English, it is not their native language. I made the choice to correct the students' spoken grammatical errors in the transcripts to enhance readability.

4. Teachers at HVIS are mostly from the United States, Canada, New Zealand, and Australia. Within the school, the teachers mostly promote student autonomy.

\section{References}

Chao, R. K. (1994). Beyond parental control and authoritarian parenting style: Understanding Chinese parenting through the cultural notion of training. Child Development, 65(4), 1111-1119.

Chao, R. K. (2000). The parenting of immigrant Chinese and European American mothers relations between parenting styles, socialization goals, and parental practices. Journal of Applied Developmental Psychology, 21(2), 233-248.

Chirkov, V., Ryan, R. M., Kim, Y., \& Kaplan, U. (2003). Differentiating autonomy from individualism and independence: A self-determination theory perspective on internalization of cultural orientations and well-being. Journal of Personality and Social Psychology, 84(1), 97-110.

Chen, W-W. (2014). The relationship between perceived parenting style, filial piety, and life satisfaction in Hong Kong. Journal of Family Psychology, 28(3), 308-314.

Chen, W-W., \& Ho, H-Z. (2012). The relation between perceived parental involvement and academic achievement: The role of Taiwanese students' academic beliefs and filial piety. International Journal of Psychology, 47(4), 315-324.

Chen, W-W., \& Wong, Y. L. (2014). What my parents make me believe in learning: The role of filial piety in Hong Kong students' motivation and academic achievement. International Journal of Psychology, 49(4), 249-256.

Chow, S. S., \& Chu, M. H. (2007). The impact of filial piety and parental involvement on academic achievement motivation in Chinese secondary school students. Asian Journal of Counseling, 14(1\&2), 91-124.

Fuligni, A. J., \& Zhang, W. (2004). Attitudes toward family obligation among adolescents in contemporary urban and rural China. Child Development, 75(1), 180-192. 
Hau, K-T., \& Salili, F. (1991). Structure and semantic differential placement of specific causes: Academic causal attributions by Chinese students in Hong Kong. International Journal of Psychology, 26(2), 175-193.

Ho, D. Y. (1994). Filial piety, authoritarian moralism, and cognitive conservatism in Chinese societies. Genetic, Social, and General Psychology Monographs, 120(3), 349-65.

Hui, E. K. P., Sun, R. C. F., Chow, S. S., \& Chu, M. H. (2011). Explaining Chinese students' academic motivation: Filial piety and self-determination. Educational Psychology, 31(3), 377-392.

Jang, H., Reeve, J., Ryan, R. M., \& Kim, A. (2009). Can self-determination theory explain what underlies the productive, satisfying learning experiences of collectivistically oriented Korean students? Journal of Educational Psychology, 101(3), 644-661.

Kim, H., \& Hoppe-Graff, S. (2001) Mothers' roles in traditional and modern Korean families: The consequences for parental practices and adolescent socialization. Asia Pacific Education Review, 2(1), 85-93.

Lew, S-C., Choi, W-Y., \& Wang, H. S. (2011). Confucian ethics and the spirit of capitalism in Korea: The significance of filial piety. Journal of East Asian Studies, 11(2), 171-196.

Li, J. (2003). The core of Confucian learning. American Psychologist, 58(2), 146147.

Li, X., Zou, H., Liu, Y., \& Zhou, Q. (2014). The relationships of family socioeconomic status, parent-adolescent conflict, and filial piety to adolescents' family functioning in mainland China. Journal of Child and Family Studies, 23(1), 29-38.

Louie, V. (2004). Compelled to excel: Immigration, education, and opportunity among Chinese Americans. Stanford, CA: Stanford University Press.

Maxwell, J. A. (2013). Qualitative research design: An interactive approach (3rd ed.). Thousand Oaks, CA: Sage.

Ng, H. Y., Griva, K., Lim, H. A., Tan, J. Y. S., \& Mahendran, R. (2016). The burden of filial piety: A qualitative study on caregiving motivations amongst family caregivers of patients with cancer in Singapore. Psychology \& Health, 31(11), 1293-1310.

Park, H-J., \& Kim, C. G. (2015). Bystander attitudes toward parents? The perceived meaning of filial piety among Koreans in Australia, New Zealand and Korea. Australasian Journal on Ageing, 35(2), E25-E29.

Pan, Y., Gauvain, M., \& Schwartz, S. J. (2013). Do parents' collectivistic tendency and attitudes toward filial piety facilitate autonomous motivation among young Chinese adolescents? Motivation and Emotion, 37(4), 701711. 
Phillipson, S., \& Phillipson, S. N. (2012). Children's cognitive ability and their academic achievement: The mediation effects of parental expectations. Asia Pacific Education Review, 13(3), 495-508.

Ryan, R. M., \& Deci, E. (2004). An overview of self-determination theory: An organismic-dialectical perspective, in Deci, E. \& Ryan, R. M. (Eds.), Handbook of self-determination research (pp. 3-33). Rochester, NY: University of Rochester Press.

Small, M. L. (2009). 'How many cases do I need?': On science and the logic of case selection in field-based research. Ethnography, 10(1), 5-38.

Yeh, K. H., \& Bedford, O. (2003). A test of the Dual Filial Piety model. Asian Journal of Social Psychology, 6(3), 215-228.

Yin, R. K. (2013). Validity and generalization in future case study evaluations. Evaluation, 19(3), 321-332.

\section{Author Contact}

Jonathan Tam: jon.cb.tam@gmail.com

University of Oxford, Wellington Square, Oxford, OX1 2JD, United Kingdom 